\title{
3 Threat analysis and attack modeling for machine-to-machine communication toward Internet of things
}

\begin{abstract}
The wide variety of Internet of thing (IoT) applications demands a secure and efficient communication channel that resists against a variety of modern attacks and fulfils application requirement. There are various IoT threats and challenges that must be addressed to make a communication secure in IoT. As growth in devices increases with their potential misuse, so there is a need to integrate security features into the available IoT algorithms and protocols. At the same time, we need to design new IoT protocols and algorithms for extended features of machine-to-machine (M2M) communication. This chapter has proposed a protocol stack that is mapped to the Transmission Control Protocol/Internet Protocol (TCP/IP) which is used for communication and connection of network devices on internet. The protocol present in a particular layer must acquire a security feature of associated layer. This chapter also elaborates on M2M communication in IoT. This chapter presents and discusses the literature survey corresponding to access control mechanism and trust management policies. From the literature survey it is observed that the existing security protocols, methods and algorithms are not suitable for IoT resource-constrained environment. The various resource-constrained devices are unable to cope up with different attacks. This chapter gives detailed analysis of attacks with its behavioral modeling. If the reader understands how attacks happen, then it will be easy to investigate solutions. From the extensive literature survey this chapter has drawn gap analysis, which gives insight into security requirements of IoT protocols and security algorithm. In addition, the chapter proposes a novel security framework that emphasizes on making secure communication layer with the help of trust management policies, distributed access control framework and privacy-aware protocols.
\end{abstract}

Keywords: Internet of things, M2M communication, threat analysis, IoT protocols, attacks

\subsection{Introduction}

The term "Internet of things" (IoT) was coined by Kevin Ashton at Procter \& Gamble in 1999 [1]. At that time, he observed that radiofrequency identification (RFID) is required to the IoT, which would help computers to handle all individual things. In the era of computation, IoT is the important buzzword that drives global market. It is the 
communication network of people, services and things. It also follows service-oriented architecture by providing service to other components of its architecture. It provides a communication channel to all varieties of devices for interaction with the help of the Internet. If we look from the architectural perspective, it is clear that it is a convergence of various technologies. The seamless integration of various technologies makes IoT more successful in global IT market. In near future, billions and trillions of devices are going to be connected over the Internet. According to a report given by global institute, considerable growth in devices of $300 \%$ has been observed in last half decade [2]. The same exponential growth is expected in near future. The objective of these connected devices is to bring betterment to human life. So, IoT is implemented in various domains such as agriculture, supply chain management, wireless sensor network (WSN) and health care. The advancement in each domain is observed in the last 5 years.

Since last few years, researchers have been trying to propose generic IoT architecture but still there is no standard architecture for IoT. Generic IoT architecture is composed of four layers, including application layer, middleware layer, network layer and perception layer [3]. According to the International Telecommunication Union (ITU), IoT architecture consists of five layers, which includes sensing layer, access layer, network layer, middleware layer and application layer [4].

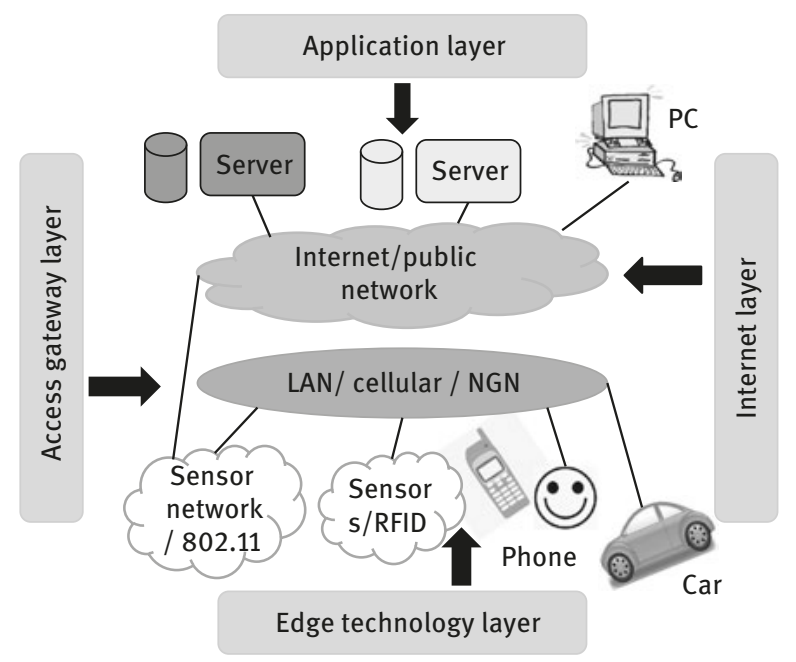

Figure 3.1: loT layered perspective.

Figure 3.1 mainly shows an architectural perspective of IoT [5]. This architecture can be viewed as a service-oriented architecture, in which each architectural component or layer provides service to other layers or component in the architecture. The edge technology layer helps to connect sensor networks, things and RFID to 
access gateway layer. This access gateway layer is a collection of various network devices and middleware which supports connectivity between Internet layer and access gateway layer. Internet layer supports Internet protocols that are required for networking. Application layer handles all types of smart applications, for example, smart transportation and smart home.

In real-time application development, the data flows from sensor devices to middleware using edge technologies like Wi-Fi and Bluetooth. The middleware is a software or hardware component that acquires data and performs basic analytics to take local decisions. This data from middleware travels toward cloud end with the help of Internet. There are various cloud service providers such as AWS, Microsoft and IBM, which provide a platform for IoT application development. Finally, this collected data is used for the analytical purpose. These analytical results help to take best decisions and for building smarter applications.

IoT is mature enough in European countries; however, there are some developing countries that are facing problems in implementing IoT [6]. At some places, the picture of IoT is still very blur. So, another term that is used as the replacement of IoT is machine-to-machine (M2M) communication. Actually, the scope of IoT is not limited to only M2M communication, rather, we can say M2M communication is part of IoT network. In M2M communication, the machines or devices are connected to each other to control, monitor and exchange data with the remote machine. M2M communication enables the capability of embedded hardware for sensing, actuation and communication. In M2M communication the network can be developed with the help of wired or wireless media to communicate with other heterogeneous types of devices. This communication allows gathering information from the edge of all enterprises and applying various ways to make a positive impact on business growth. M2M communication is a subset of IoT. In M2M communication participating devices are independent of Internet connection. In M2M communication there are limited options for integration in the system because participating devices need to match communication standards. In the IoT, there are unlimited options for integration but it requires a solution that efficiently integrates all system components [7].

TCP/IP protocol stack gives a practical orientation of real-time communication. This suite consists of various protocols that work at different layers but the problem with the existing protocol is that it is not suitable for IoT [8]. IoT network consists of resource-constrained devices. Hence, IoT is in need of a protocol stack that accommodates lightweight protocols of IoT.

\subsubsection{Various protocols used in loT at all layers}

IoT follows service-oriented architecture, which means one component of architecture provides service to another layer of architecture. If we look it as a layered architecture, 
then one layer provides services to other layer. This task is beautifully carried out with the help of protocols. Figure 3.2 shows protocols used at different IoT layers.

\begin{tabular}{|c|c|}
\hline $\begin{array}{l}\text { Application layer: } \\
\text { HTTP COAP MQTT XMPP } \\
\text { RESTFUL }\end{array}$ & DDS JSON \\
\hline $\begin{array}{l}\text { Transport layer: } \\
\text { TCP UDP TLS DTLS }\end{array}$ & \\
\hline $\begin{array}{l}\text { Network layer } \\
\text { IPv4 IPv6 6LoWPAN RPL }\end{array}$ & \\
\hline $\begin{array}{l}\text { Link layer } \\
\text { 802.3-Ethernet 802.11-WiFi } \\
\text { 2G/3G/LTE-cellular }\end{array}$ & 802.16-WiMax \\
\hline
\end{tabular}

Figure 3.2: Protocols used in loT at all layers.

\section{Link layer}

Link layer decides to transfer data physically over network's physical layer or medium, for example, radio wave, coaxial cable or copper wire. The scope of link layer is a local network, a connection to which the host is attached. Data packets are exchanged over link layer by host using link-layer protocols. Linklayer protocols in context of IoT are 802.3- Ethernet, 802.11-WiFi, 802.16WiMax and 2G/3G/LTE-cellular.

2. Network layer

This layer is responsible for sending of IP datagram from the source network to the destination network. Protocols used in this layer are IPv4, IPv6, and 6LoWPAN.

\section{Transport layer}

The protocols in this layer provide end-to-end message transfer capability. Protocols used in this layer are TCP, UDP, TLS and DTLS.

\section{Application layer}

Protocols in this layer define how application interface with lower layer protocols to send the data over the network. Protocols used in this layer are HTTP, CoAP, MQTT, XMPP, and DDS.

\subsection{Motivation}

IoT promises a key role for information transaction and medium, management and computing since the beginning of the twenty-first century. As discussed in the above section, it is clear that IoT is open to the Internet and it is obvious that it is 
open to the attacker to attack the system. Consider any application like health care, military, smart home, smart industries and so on. These industries use IoT-enabled systems. Now, in these systems, sensors sense an environmental parameter and pass data to the next IoT component and final destination of data is a cloud. In the above scenario, data travels from the sensor node to cloud environment, and in transit the data is open in Internet. Attackers try to attack the system and try to collapse the system. In order to provide security to the whole IoT network, it is required to analyze every type of attacks that can happen in IoT. Purely, security to data is not sufficient; we need to provide security at each layer and to each component of IoT architecture. IoT consists of resource-constrained devices for which we cannot use existing security protocols, algorithms and policies. In order to maintain security of each solution against every attack, we need to study behavioral modeling of each exposed attack. The objective of this chapter is to understand security in IoT as it is a fundamental pillar of IoT ecosystem. At the same time, it is required to analyze behavioral modeling of most possible attacks to provide a solution set in near future.

\subsection{Threat analysis}

\subsubsection{Threats and attacks at various layers}

Table 3.1 lists the threats and attacks at various layers.

A threat is something that may or may not happen, but has the capability to cause serious damage to network and data as well. Threats can lead to attacks on computer systems, networks and more. Security threats and vulnerabilities of the M2M communication in the IoTs are explored in this section.

\subsubsection{Threat Analysis}

Security and privacy is always a major concern when data and resources are open to Internet. In order to develop security solution it is required to analyze the behavior of each and every aspect of threats. Security threats have been explained in the context of M2M communication.

\section{Cloning of thing}

Many times an untrusted device manufacturer creates a clone device that is having identical security configuration, link-layer properties and unique identification of things of real one. To attract customers, these cloned faulty devices are sold at 
Table 3.1: Threats and attacks at various layers.

\begin{tabular}{|c|c|c|}
\hline Layer & Threats & Attacks \\
\hline Application layer & $\begin{array}{ll}\text { - } & \text { Warms and virus } \\
- & \text { Trojan horse } \\
- & \text { Buffer overflow } \\
- & \text { APP/OS weakness. } \\
- & \text { Identity theft and } \\
& \text { unsecured end devices } \\
- & \text { Insufficient patching } \\
& \text { and testing } \\
- & \text { Multilayer data manage- } \\
& \text { ment and security } \\
- & \text { Phishing } \\
- & \text { Ransomware } \\
- & \text { IoT botnets }\end{array}$ & $\begin{array}{ll}\text { - } & \text { mpersonation attack } \\
& \text { or clone attack } \\
\text { - } & \text { Man in middle attack } \\
\text { - } & \text { DoS attack } \\
\text { - } & \text { Malware } \\
\text { - } & \text { Phishing } \\
\text { - } & \text { DDoS attack } \\
\text { - } & \text { SQL injections } \\
\text { - } & \text { XMAS attacks }\end{array}$ \\
\hline Presentation layers & $\begin{array}{ll}\text { - } & \text { Viruses } \\
\text { - } & \text { Wormwares }\end{array}$ & - \\
\hline Session layers & $\begin{array}{ll}\text { - } & \text { Personal information } \\
& \text { retrieval } \\
\text { - } & \text { Root privilege access } \\
\text { - } & \text { Net bios } \\
\text { - } & \text { DoS }\end{array}$ & - \\
\hline Transport layer & $\begin{array}{ll}- & \text { Port scanning } \\
- & \text { TCP sync flooding } \\
- & \text { UDP flooding }\end{array}$ & $\begin{array}{l}- \text { DOS } \\
-\quad \text { DDOS }\end{array}$ \\
\hline Network Layer & $\begin{array}{ll}\text { - } & \text { Sniffing } \\
\text { - } & \text { IP alteration } \\
\text { - } & \text { DHCP attack } \\
\text { - } & \text { Phishing }\end{array}$ & $\begin{array}{l}\text { - } \text { Man in the middle attack } \\
\text { - } \text { ICMP attack } \\
\text { - } \text { Sybil attack } \\
\text { - } \text { DoS attack } \\
\text { - } \quad \text { Sinkhole attack }\end{array}$ \\
\hline Data link layer & $\begin{array}{ll}\text { - } & \text { ARP attack } \\
\text { - } & \text { MAC address alteration } \\
\text { - } & \text { MAC flooding }\end{array}$ & $\begin{array}{l}\text { - } \quad \text { ARP spoofing } \\
\text { - } \quad \text { Sniffing } \\
\text { - } \quad \text { MAC flooding attacks }\end{array}$ \\
\hline Physical layer & $\begin{array}{l}\text { - Cable disconnected } \\
\text { - Cloning of things } \\
\text { - Unauthorized access to } \\
\text { tags } \\
\text { - Tag cloning }\end{array}$ & $\begin{array}{l}\text { - Passive sniffing over a } \\
\text { media }\end{array}$ \\
\hline
\end{tabular}


lower price in the market. With the help of these devices, an attacker can hack original devices or degrade the performance of genuine devices. With cloned things, the manufacturer can observe the data traveling in the network and may perform a Sybil attack and node replication attack. A manufacturer can implement a backdoor for any confidential application. In the RFID system, tag cloning is easily possible because of weak authentication mechanism [9].

\section{Commissioning of a thing}

At the time of commissioning of things, things can be vulnerable to eavesdropping attack. Mostly configuration settings, security parameters and keying materials may be compromised through wireless medium. The attacker might be able to recover the secret key after obtaining keying material, thereby the authenticity and confidentiality may be compromised. If communication channels are not properly protected, M2M communication may eavesdrop or even session key can be compromised because of a long period of usage without key renewal or updates.

\section{Malicious replacement of things}

Sometimes attacker adds a new node in the network of participating node and assigned node identification information. The replaced node is involved in malicious activities. Sometimes it is possible that, in order to save the cost and increase profit from the system, the lower quality sensors are placed in the network. Attacker finds out such low-quality nodes and targets them for malicious activities, Due to this attack, the performance of the system is hampered. At the same time attacker can change the direction of packet toward malicious server enabling an attacker to monitor network activities [10].

\section{Unreliable communication}

If we use an unreliable communication link it raises the question of data integrity. The attacker can target this unreliable communication medium as a target and can perform a man in the middle attack. The unreliable communication link does not guarantee the successful delivery of the message. If packets are not delivered to destination, retransmission of packet takes place, which leads to dropping down the performance of the system. Due to multiple retransmissions, the time required to reach destination also increases, which affects the throughput of the system [11]. 


\section{Resource constraints}

The IoT network is mostly filled with embedded computing devices that appear to be resource constraint. The resource constraints are not only applied to the memory and processing capability but also to low bandwidth utilization, which leads to constraints on the network interface. Due to the resource-constrained devices, IoT system is vulnerable to security attacks.

\section{a. Limited computing power and memory}

These embedded devices require to possess sufficient amount of computational power with which they can complete the allocated task. To improve the lifetime of embedded devices, these devices should be designed in such a way that they can perform the required set of operations with minimum computational efficiency as it has limited memory and storage. For resource-constrained devices, we need to analyze bare minimum features that will be integrated into embedded devices. The researcher needs to develop lightweight security protocols that are complex to break by considering crucial security measures. There is always a trade-off between processing power and the memory requirement of security algorithm. For these protocols, we need to leave enough space for security software that strongly resists security threats [12].

\section{b. Limited battery}

IoT is a network of connected devices. The idea here is to connect everything to everything to collect data and obtain analytical results from that data to make an intelligent system. Most of the time the remotely placed devices are battery powered. Therefore, researchers are working on ideas/mechanism to increase the lifetime of the battery. There are lots of parameters that decide the lifetime of the battery. Security protocol, mathematical operation and network protocol are the key parameters that drain the major portion of the battery. The complex security protocols lead to battery drain, due to which lifetime of the device decreases. Hence, there is a need for lightweight security protocols with minimum computation and memory that does not harm the lifetime of devices [13].

\section{Identity theft and unsecured end devices}

In IoT, security plays an important role in real-time scenarios. In IoT, we need to take care of devices, especially those devices that hold important financial and private information. In some IoT systems, these devices are made secure with different security mechanism. But all IoT devices are not secure enough to prevent identity theft and security breaches, for example, there are a variety of vendors 
selling a smartwatch but only 50\% smartwatches allow its users to set authentication password. There are various enterprises that do not bother about vulnerabilities within IoT devices. The available security mechanisms try to prevent attack; however, the attacker hacks the data center or network. Due to this type of theft, impersonation attack or clone attacks are possible [14].

\section{Privacy threat}

In IoT, there are some applications that carry user's sensitive data. It is required to protect that data from being exposed to IoT environment. It is a major point of concern if devices carry health-related data. The services provided by these connected devices offer better human life but at the same time, the privacy of the user should not be compromised. The tracking of objects' location and its usage may lead to increased privacy risk to the end users. When information is passed inside IoT systems only, the attacker may infer the information by performing analytical operation and inferred data can predict the behavioral pattern of user's interest and such information will be sold for marketing purpose.

\section{Insufficient patching and testing}

Insufficient patching is the most common and the biggest problem faced by the IoT ecosystem. The problem of patching is mostly ignored by the system supervisor. The outdated devices may have some software issues, incompatibility with other devices, set of protocols used or it may contain some bugs or vulnerabilities. So, to break the security of the system, attacker may use these vulnerabilities as the door to open into systems. The patching is required to secure the system from the above-mentioned vulnerabilities. Now, it is important to monitor and test each updated path to maintain stability of the system. Insufficient patching and testing also give way to the attacker to enter into a system that leads to cloning attack or malware [15].

\section{Multilayer data management and security}

The IT industry is moving rapidly, so in a real-time scenario, it becomes very much necessary to adopt changes by deploying new technologies like cloud, big data and IoT. This deployment of new technology is more important than securing the infrastructure and network. As IoT drives the global market including financial organizations [16], it should not compromise with security threats. By considering all aspects of infrastructure, security should be placed at the central point of 
investment. For any application development, we need to provide security at each and every layer of our network. The layers at which security is important are (1) end devices, (2) software configurations, (3) communication framework or channel and (4) web cloud and mobile environment.

\section{Phishing}

It is a kind of social engineering attack in which an attacker steals user's sensitive data like login credential or credit card information. It is not a new type of attack but despite growing awareness about this attack, organizations are unable to provide full resistance against sophisticated social engineering attack. This attack has a variety of results [17].

\section{Ransomware}

Ransomware is a kind of malicious software in which attacker threatens the victim to publish victim's sensitive information or purposefully block access to its resources until a ransom is paid to the attacker. This attack is performed through Trojan. This Trojan file hides behind the legitimate file and is sent to victims as an email attachment. When the victim opens this file, the malware takes the ownership rights of the victim's resources. For relieving access to victim, attacker demands ransom. The example of ransomware is "WannaCry" ransomware, which automatically travels in the network without any interaction with victims. The windows user is mostly affected by this attack. The evolution of this attack is still in progress. Generally, a target for attacker is a high-end server because it carries a massive amount of sensitive data [18]. The windows user mostly affected by this attack.

\section{loT botnet}

The distributed denial of service (DDOS) performed on Dyn proves the potential of DDoS attack in IoT. This happens because of the lack of embedded security and fewer security considerations for participating devices. The botnet is the most widely used weapon to attack IoT devices. Botnet means the computer that is controlled remotely. The access to devices is gained by injecting malware inside the devices. These infected devices communicated with other participating nodes in the network or server and wait for an instruction from the attacker [19]. 


\subsection{Attack modeling}

The objective of this section is to provide information about how attacks are performed. This helps the researcher to work on mitigation technique to prevent the attack or to control the damage.

1) Sybil attack

In IoT, each individual device carries its own identity. In this attack, malicious nodes communicate with other nodes via separate communication link. For identity establishment, the attacker creates duplicate identity of the legitimate node and hides behind that node. In this attack, the attacker is present at multiple places at the same time. This can be done by identity spoofing [20].

In the given Figure 3.3, device 1 gets access from server after authentication. Then the attacker gets the id proof of device 1 from server and starts communicating with the other device pretending to be device 1 communicating with device 2 . In this process, the id proof of device 1 gets compromised and is used by the attacker to retrieve data from device 2 .

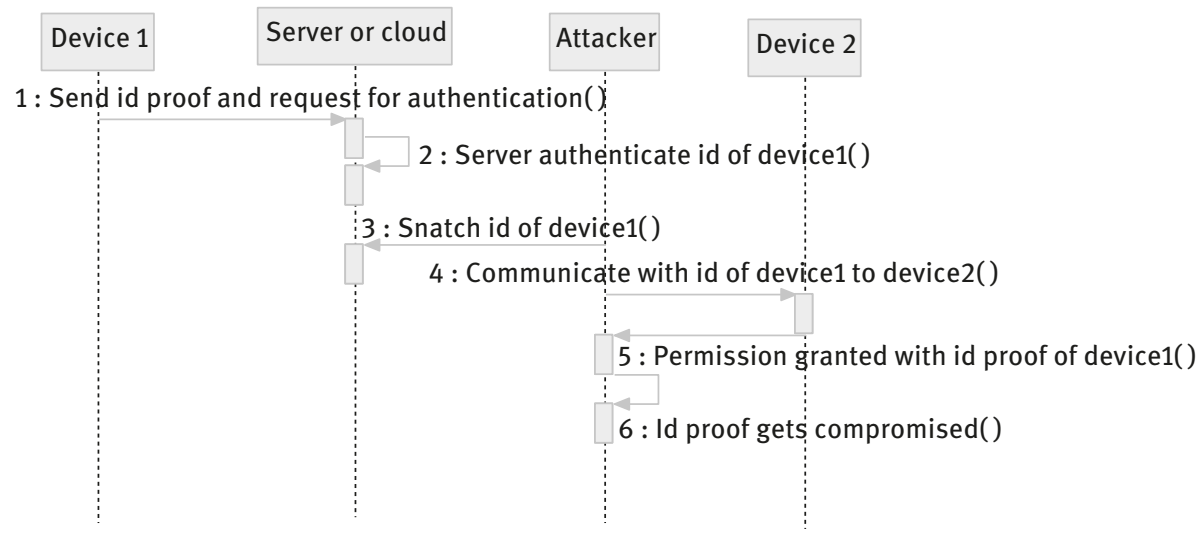

Figure 3.3: Sybil attack.

2) Data integrity attack

In this type of attack, the data is monitored or altered when it travels through the network. The attacker changes the contents of the data packet by injecting false information into the packet. By performing this attack, attacker increases impurity in the sensor data and by doing so he creates an obstacle in victim's research. We can say that it is a kind of DoS attack. The impure data gives poor decisions. The attacker adds impurities in victim's database that are in acceptable range. So, it is very difficult for the victim to find out and separate impurities in the dataset. Various practical approaches are presented to prevent this attack [21]. 
Figure 3.4 shows how data integrity attack happens. In data integrity attack, attacker targets any legitimate node of the network. Then attacker injects malicious code into that node. This infected node acts as a malicious node. An attacker can change the code of a malicious node; it alters data that is transmitted by the malicious node. This faulty data will be transmitted to all other nodes in the network. The communication between all these nodes has been represented in Figure 3.4.

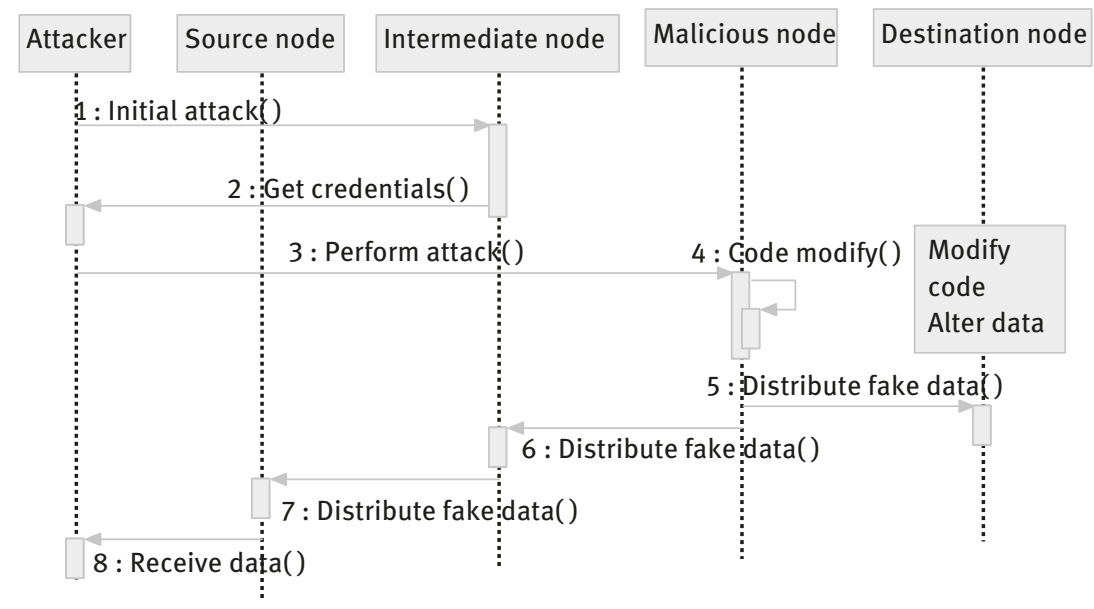

Figure 3.4: Data integrity attack.

3) Node replication attack

This is an application-independent attack. First, attacker captures a single legitimate node and gathers information like secret credentials, code and cryptographic material, and if required attacker can modify code and change the behavior of node. Further attacker replicates the same image to other node and deploys these replicated nodes in the environment. This type of attack is very destructive. The general-purpose authentication and security methods cope up with this attack [22].

The behavior of node replication attack is represented in Figure 3.5. Various entities participate in this communication. The attacker initiates the attack and targets any legitimate node of the network. Before the attack, attackers analyze all unsecured roots through which they can enter into the network. Once they are able to manage legitimate node then attacker collects all the secret information of node such as credentials and secret keys. Once an attacker gets this information of the legitimate node, the information will be modified. According to the attacker's requirement, the same information will be implanted in node memory. After that these infected nodes act according to the attacker's instructions. In this way attacker can make the target network unstable. 


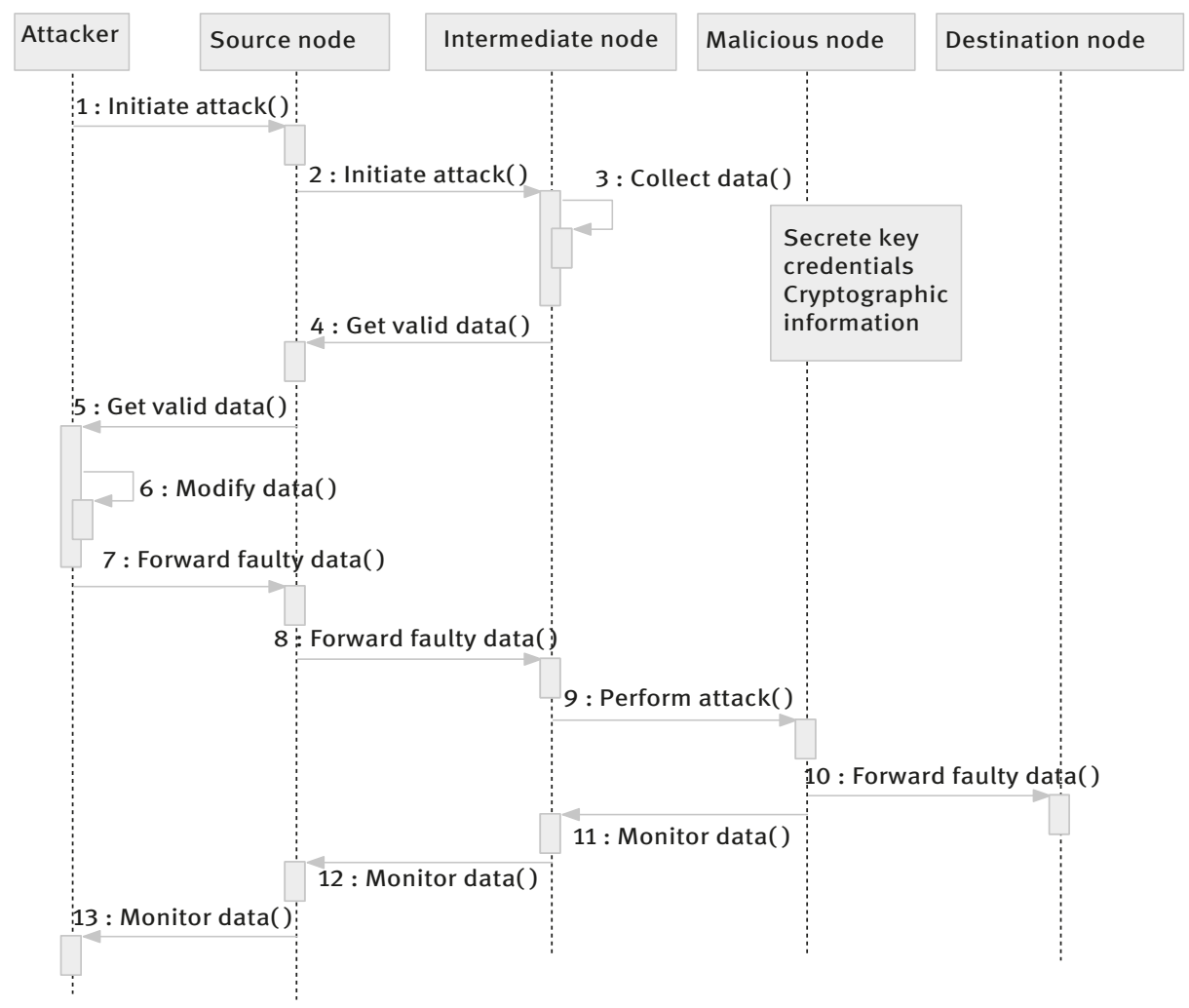

Figure 3.5: Node replication attack.

\section{4) DoS attack}

Denial of service (DoS) attack makes service unavailable. The attacker makes an attempt to prevent users getting services or access to resources. DoS is difficult to perform but it is dangerous. The attacker continuously sends request packets to the server. If the server is unable to handle these large number of requests, then it results in server unavailability [23].

In Figure 3.6, device 1 is able to get services from server after its identity is verified. After that device 1 gives encrypted data to server, which is retrieved by the intended device. Then device 2 gets authenticated from server and tries to access encrypted data stored on server by device 1. At the same time attacker uploads large file on server or continuously gives data to server, thereby preventing device 2 from getting its requested encrypted file from server leading to buffer overflow, due to which server is unable to give service to device 2. In this way, DoS attack is done by the attacker. 


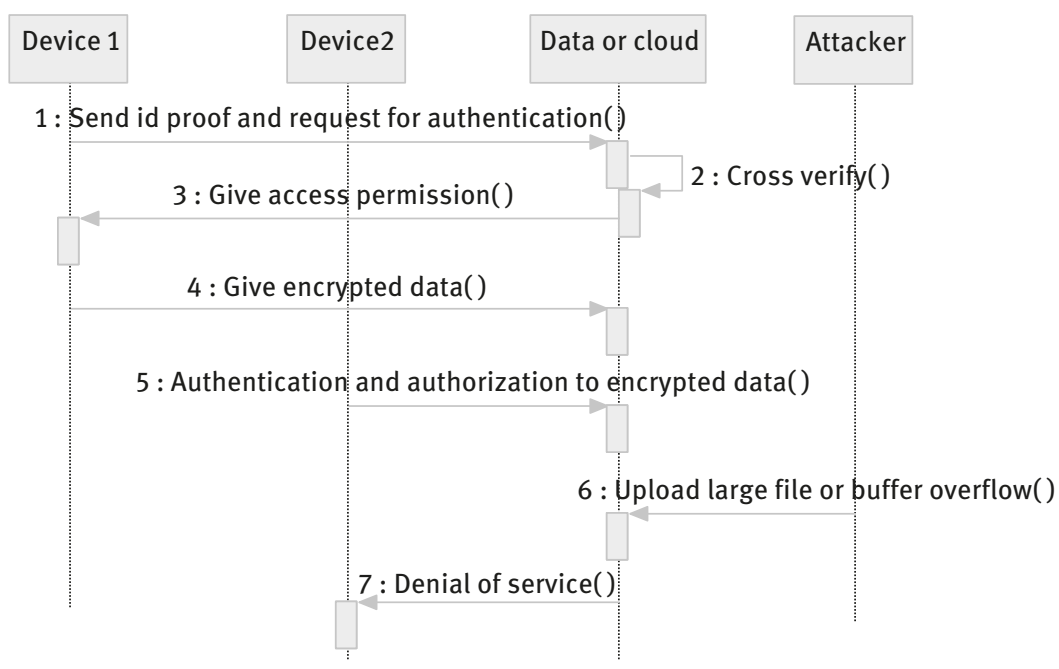

Figure 3.6: Denial of service attack.

5) Replay attack

It is a type of network attack in which data transmission on authenticated channel is observed by using legitimate user credentials and repeatedly transfers data to the destination node. This attack can be done by either originator of message or man in the middle. It is also a kind of man in the middle attack [20, 24].

The sequence diagram of replay attack is represented in Figure 3.7. In this type of attack, attacker attacks legitimate nodes of the network and collects all the information from the intermediate node. An identity is assigned to the compromised node. The attackers spread wrong data in the network or turn traffic of malicious node toward the compromised server. Valid data transmitted is repeated either by malicious node or source node. Malicious node can also intercept and modify data.

6) Man in middle attack

In this attack, attackers try to gain control of the communication link that connects the endpoints. This attack leads to compromised data confidentiality and data integrity. It is also referred as bucket brigade attack or fire brigade attack. There are various versions of this attack. In this type of attack, attacker can alter the data while two parties are communicating and we believe that they are directly communicating [25].

A public key cryptosystem is vulnerable as shown in Figure 3.8. For communication between two devices, key exchange is required in public key cryptosystems. When device A wants to communicate with device $B$, device A requests to device B for its B's public key. An attacker intercepts its request and sends its own public 


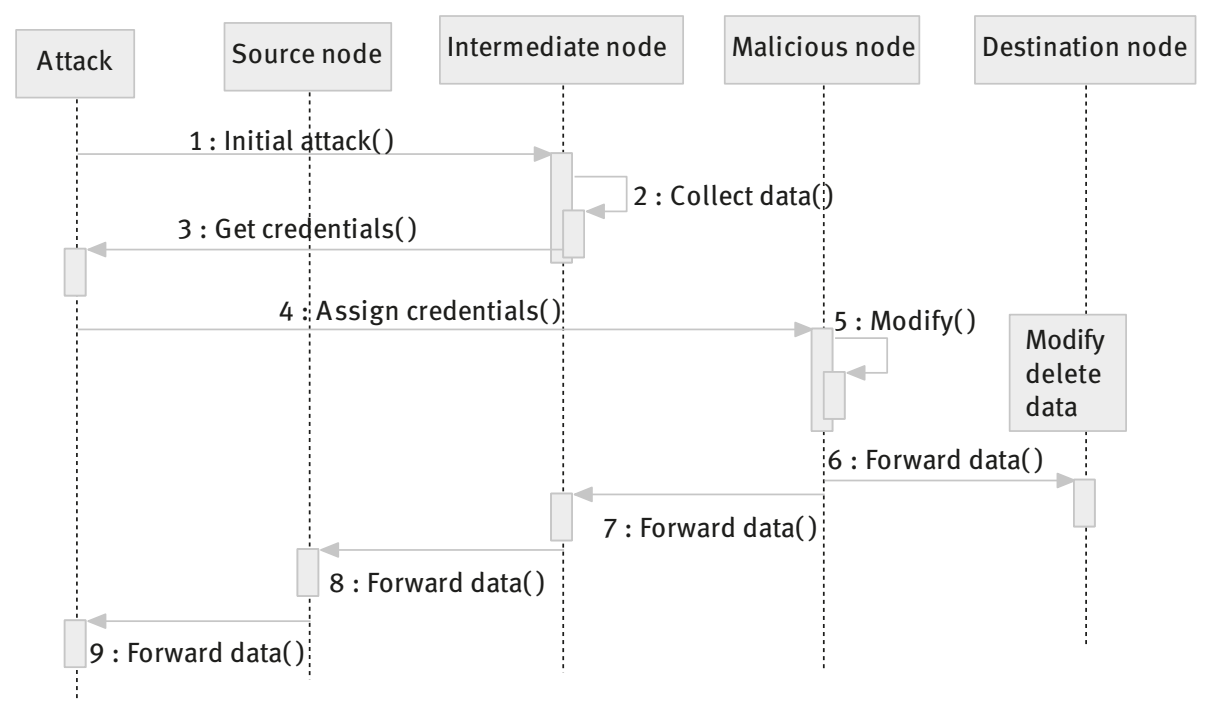

Figure 3.7: Replay attack.

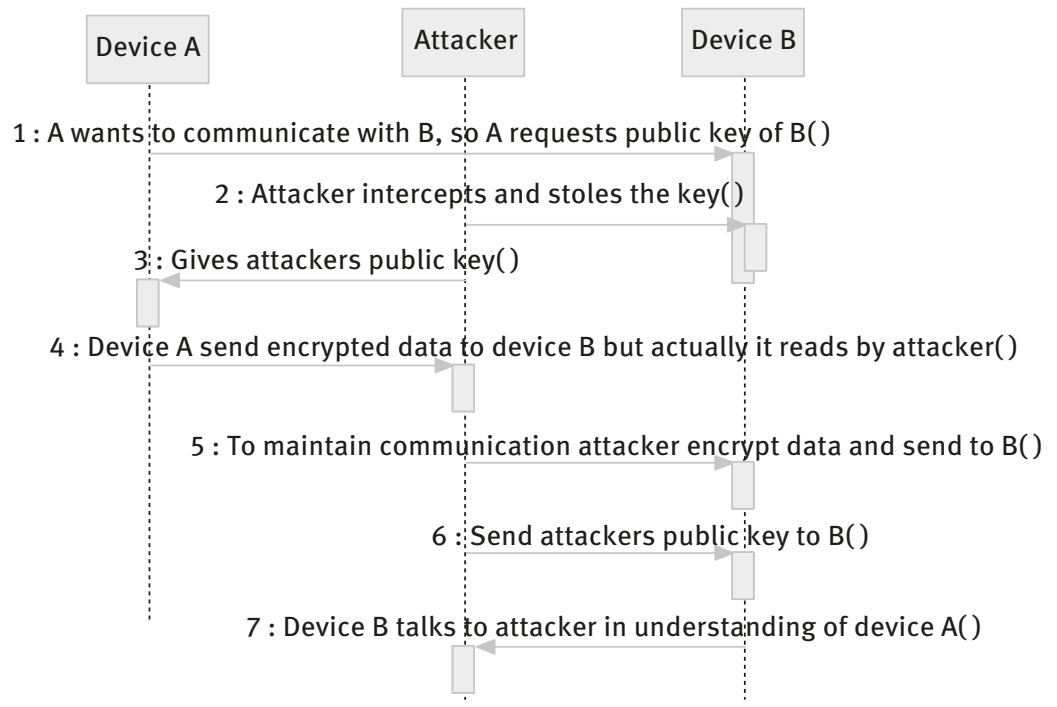

Figure 3.8: Man in middle attack.

key to device A. So whatever sent by device A to device B is intercept and read by an attacker. For maintaining communication, attacker re-encrypts this data by device B's public key and sends it to device B. Attacker also sends its own public key as A’s public key to device B. 


\section{7) Sniffing}

It is a program that monitors data traveling over the network. This sniffing is required for monitor-authenticated network as well as for stealing network information. These unauthorized sniffers are very much harmful because it is very difficult to identify sniffer-affected nodes and can be inserted at any node of network. This makes sniffing attack more popular in attacker community [26].

In Figure 3.9, devices 1 and 2 communicate with each other via server. The encrypted data is stored and is accessed by device 2 which is monitored or sniffed by the attacker. Finally, attacker gets the intended data that is sniffed by device 1 during the process of storing in server. Then attacker makes some changes into the encrypted data. Thus, device 2 receives corrupted data.

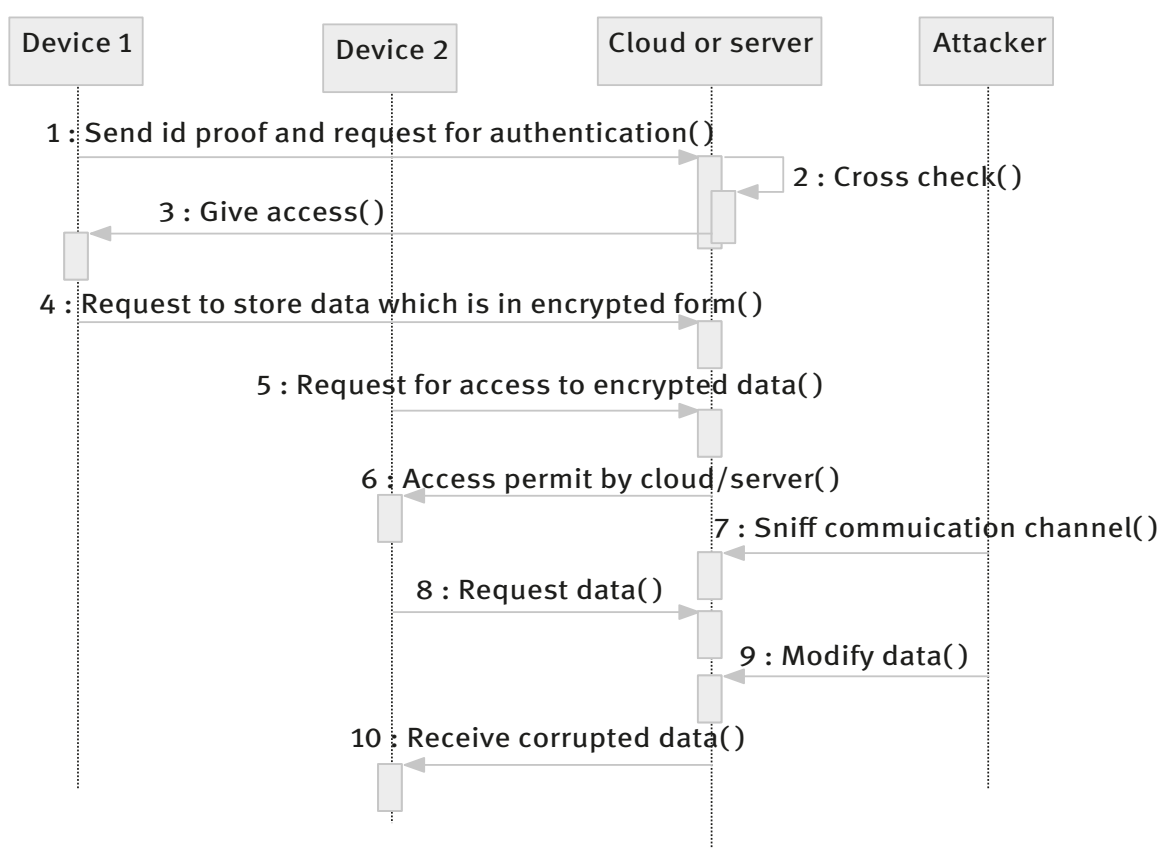

Figure 3.9: Sniffing.

8) Ransomware

It is a kind of malware that restricts the victim to access its own resources like important files of computers and demands for money to remove restriction on resources. There are two very common mediums through which these ransomwares enter the system, which are phishing email and USB drives that contain malicious software or files [27].

In Figure 3.10, attacker enters into the system through email attachment files, external hard disk drives or USBs, which causes worms or viruses to enter into the 


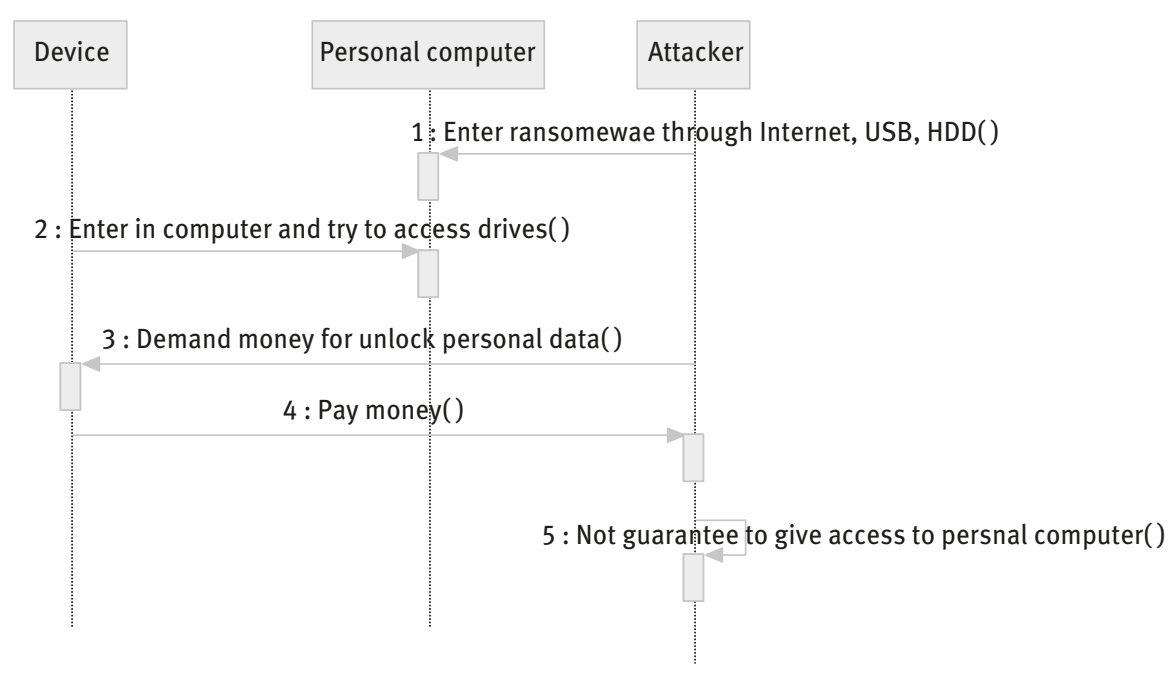

Figure 3.10: Ransomware attack.

system and blocks the personal data and drives of the device. When the device tries to access the personal data, then attacker demands money for unlocking of personal files. If device makes payment then also attacker does not guarantee to give access to its personal data or drives. As per Figure 3.10 attacker sends email with attachment and victim opens that attachment. Now, the malware restricts the victim from accessing its own resources like important files of computers and demands for ransom. Once payment is done then only attacker releases the victim's resources.

\subsection{Literature survey}

As outstanding research has been carried out in the field of IoT, however, there are still various vulnerabilities present, which makes IoT insecure. As a result of this, attackers have invented so many attacks on IoT even before IoT could mature in the IT industry. So there is a need to study the various leading attacks and threat analysis in IoT. The large scale of heterogeneous and resource-constrained devices with lack of uniformity makes it challenging to provide security in IoT environment.

Kaur and Singh [28], and Reed [29] classify attacks on the basis of the OSI layer. The variety of security attacks on RFID system have been addressed in [30]. The variety of attacks on RFID systems along with possible solutions have been proposed in [31]. General categorization of attacks has been proposed in [32]. This paper classifies attacks by considering properties and target layer into four categories, that is, encryption attack, software attack, network attack and physical attack. According to the literature survey, possible categorizations of attacks in IoT are shown in Figure 3.11. 


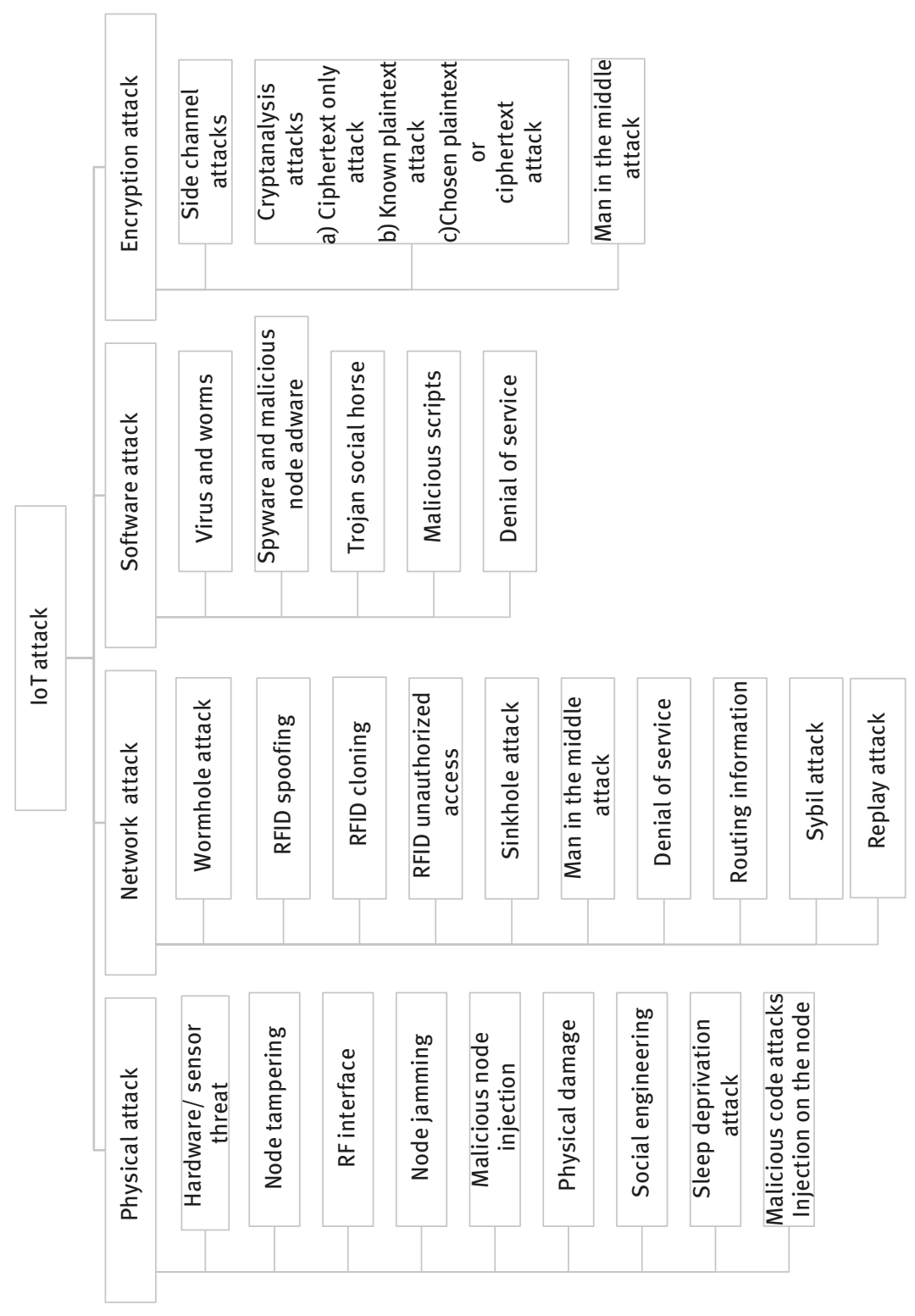

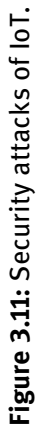


Barki [33] emphasizes on M2M research and development works, which mainly addresses security, threats and vulnerabilities that occur in M2M communications. Sicari et al. [34] give an overview of research challenges with possible solution methodologies to make IoT ecosystem secure. There are various security issues but major ones are identified in eight categories: (1) proper authentication mechanism, (2) confidentiality, (3) access control policies, (4) trust-related issues, (5) policy enforcement, (6) mobile security, (7) security of middleware and (8) privacy policies. Firdous et al. [35] explain the MQTT threat model and they evaluate the performance of DoS attack that targets MQTT brokers.

In real-time scenario, millions and billions of devices are communicating with each other. Sometimes devices carry data from users' personal space and travel over the Internet. Therefore, there are more chances that the privacy of data gets compromised. In [36], the various privacy policies for different data have been proposed to protect user's privacy. Each privacy zone is mapped with the contextbased method and is verified by Home Security Hub. This process is done before acceptance of joining or rejoining of requests to protect user data against unauthorized data sharing. However, the chances of locating smart devices directly bypassing the hub are not considered.

Que and Ma [37] propose a novel methodology for mutual authentication and key establishment scheme for the M2M communications, which uses 6LoWPAN networks. The session key will be established to authenticate each device with remote server in 6LoWPAN networks. This scheme also prevents various malicious attacks like Sybil attacks, replay attacks, MITM attacks and impersonation attacks. This scheme also incurs less computational overhead and transmission overhead.

Lin et al. [38] propose a local authentication and access control scheme, which is designed for verification of access rights and user privileges in M2M communication. Device heterogeneity is also supported by this scheme. In order to improve the scheme, a novel SOC (securely outsourcing computation) algorithm has been proposed. This algorithm offloads the computational cost from M2M devices to the user equipment. This algorithm is also energy efficient, and also satisfies the security criteria of user anonymity, secure key agreement, mutual authentication and SOC.

Mahalle et al. [39, 40] have designed an identity establishment and capabilitybased access control protocol. This scheme uses elliptical curve cryptography for security. This scheme also supports protection from replay attack, man in middle attack and DoS attack.

Esfahani et al. [41] have proposed a hash-based lightweight authentication mechanism, which uses XOR operations in M2M communications. The proposed methodology incurs low computational cost, and at the same time it removes the burden of communication and storage overhead for achieving session key agreement and mutual authentication, and holds a device's identity confidential. This scheme is also attack resistant to modification attack, replay attack, impersonation attack and man in the middle attack. 
Chen et al. [42] propose a security gateway application that will help to improve gateway application of ITU-TM2M service layer. This ITU-TM2M service layer is a layer of IoT's reference model. This application also provides a secure end-to-end M2M message delivery and key exchange generation functions. This application supports mutual authentication and symmetric cryptographic negotiation function which is of lightweight. This application is resistant to data privacy attack and relay attack. It also prevents key guessing attack and undetectable online key guessing attack.

Mahalle et al. [43] present key challenges, design constraints and framework for identity management in IoT. Matrix required for identity is explained in this paper.

Dey et al. [44] cover applications of big data generated by IoT and how to manage big data generated with all sensors and embedded devices. Applications like smart cities, industrial IoT, health care, robotic sensor networks, and smart irrigation and green cities are described properly in their contribution. Security for electronic patient record is explained in detail and the system also tested against attacks. Security issues for big data generated by IoT and attacks like side channel attack are also discussed.

Dey and coworkers [44-47] give an overview and implementation details of IoT-based wireless body area network (WBAN) in health care. Layerwise WBAN architecture, challenges and required security are explained in [45]. IoT brings extreme changes in the field of health-care technology. Bhatt et al. [46] describe challenges in smart health-care systems and possible vulnerabilities in e-health context. They also introduced energy-efficient health-care systems. Privacy, trust and security issues are also addressed. IoT architecture is explained in [47]. They have also explained IoT applications in health care.

Hassanien et al. [48] address the problems related to the Internet of medical things. They have provided solutions to challenges of medical big data and also recent techniques for classification of medical big data and machine learning. Privacy of data and security analysis is also presented for the Internet of medical things.

There is tremendous growth in digital communication and massive growth in digitization of data from the last few years and it is continuously going to increase as there is an evolution in IoT. Sarowar et al. [49] gave examples of the mechanism of messaging in M2M communication, in mobile computing and in sensors. They have given new techniques for spoofing detection of location, new efficient processing ideas and clustering tools for next generation are also presented.

\subsection{Evaluation of related work}

This section gives the comprehensive analysis of various attacks. In the following table, the detailed analysis of some outstanding research papers has been done on the basis of the attack-resistant mechanism proposed. 
In Table 3.2, Sybil attack, man in the middle attack, DDOS attack, replay attack and identity attacks are taken into consideration because these attacks are very common in today's connected world and is widely performed by a hacker. There is a need of distributed framework that considers all dynamic aspects of security in IoT ecosystem. This framework should be lightweight, scalable, adaptive and simple. There are many methods proposed by the researcher to resist the abovementioned attacks. So, we need to design the framework in such a way that it accommodates Identity establishment, device authentication methods, lightweight key management schemes, access control policies and protects privileges of the user. Table 3.2 also represents solutions for security and also tells highlights which solution is resistant to which attacks. The gap analysis is also presented in this table. We can observe that there is a need for the security framework that protects the system against various attacks. Most of the researchers try to make the authentication process more secure with optimization in computational cost and memory. All proposed methodologies or algorithms fulfill the requirements of resource constraints and is mostly applicable to the device-to-device communication.

\subsection{Proposed work}

Looking at various threats and in the sequel several attacks in resourceconstrained IoT and M2M communication, security is of prime concern. Therefore, we need access to control solution, trust management scheme and privacyenabled communication scheme. In this view, secure communication framework is shown in Figure 3.12.

The proposed architecture is divided into three layers:

1. Device layer

2. Secure communication layer

3. Application layer

\subsubsection{Device layer}

Here devices are with network capabilities ranging from high-end devices such as mainframes to simple sensors that have communication capability. It is very difficult to deal with resource-constrained devices. This layer may include sensors, actuators, WSN and other things. It is required to enable capability of devices in terms of computing, communication and sensing ability. The data collection node helps to aggregate data and at the same time plays a vital role in device management task at physical layer. The quality of data determines the quality of result 


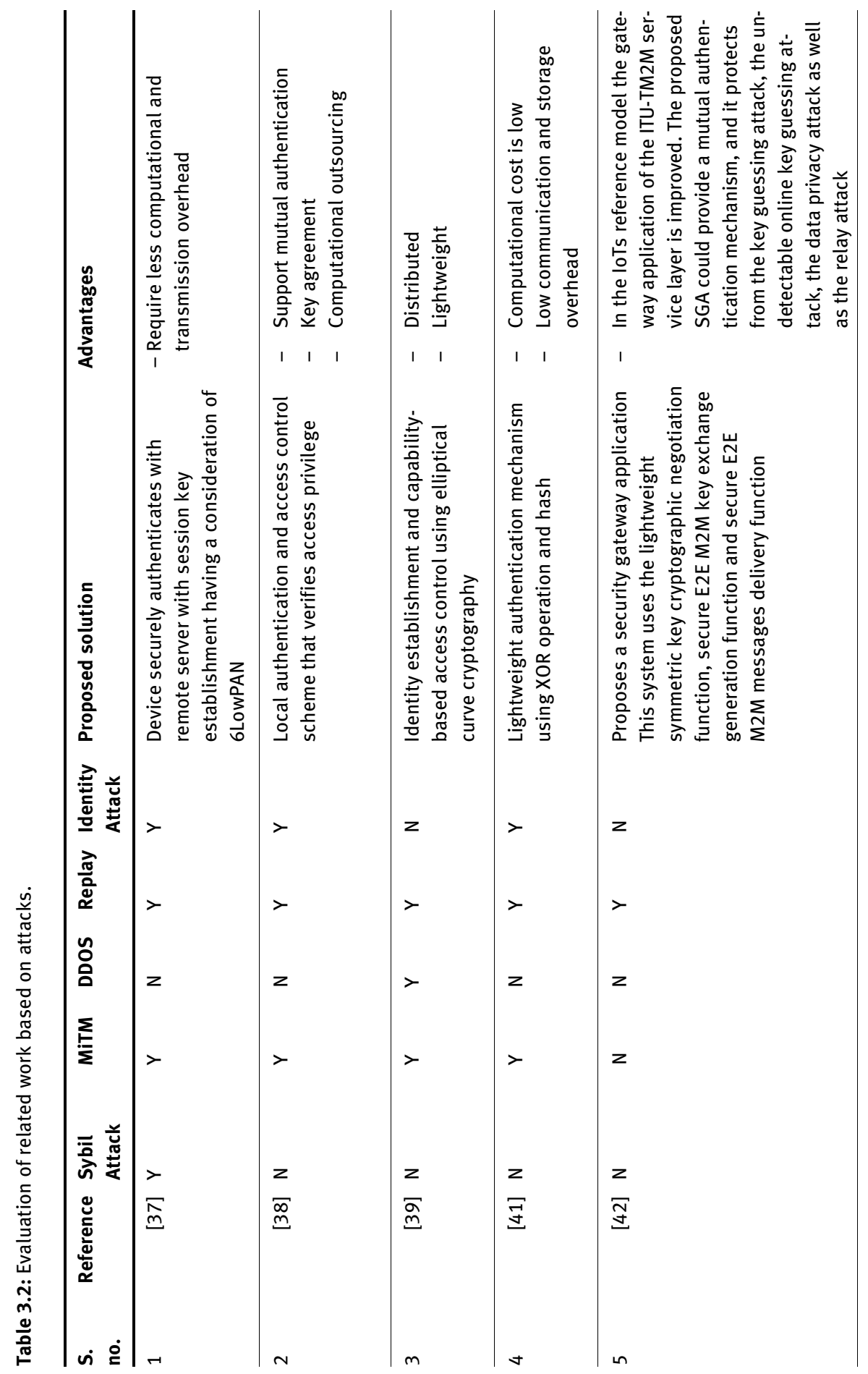




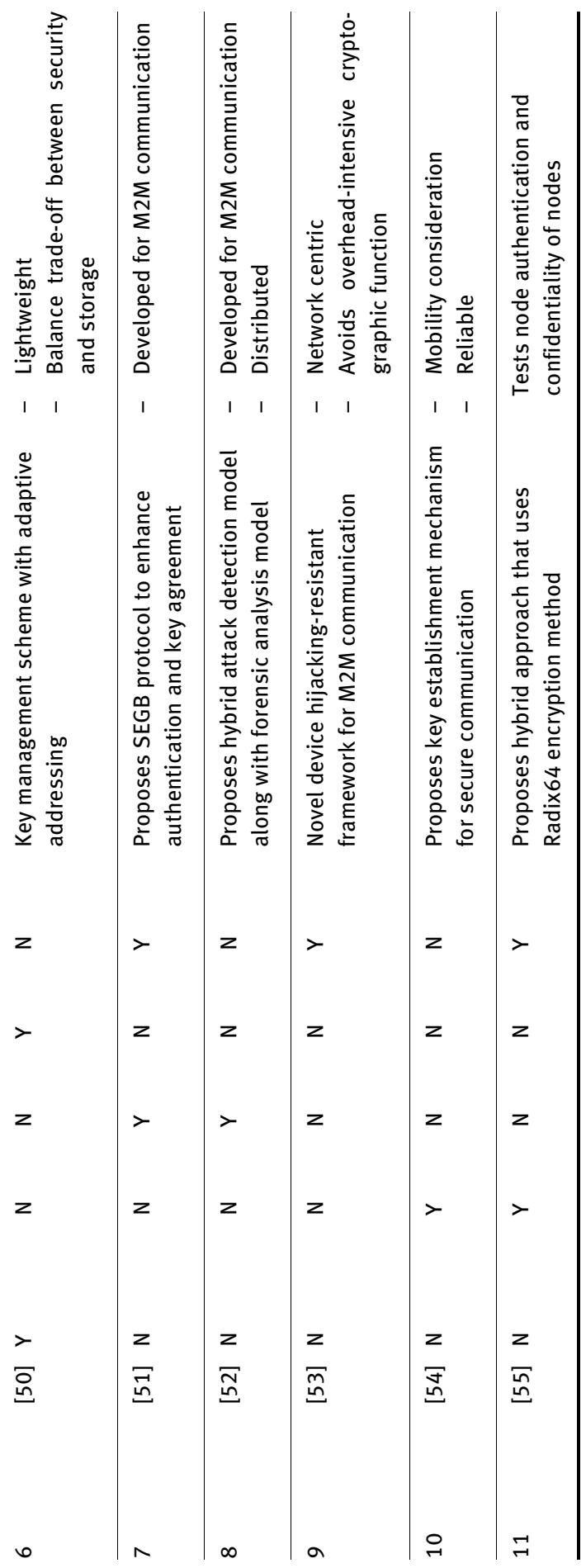




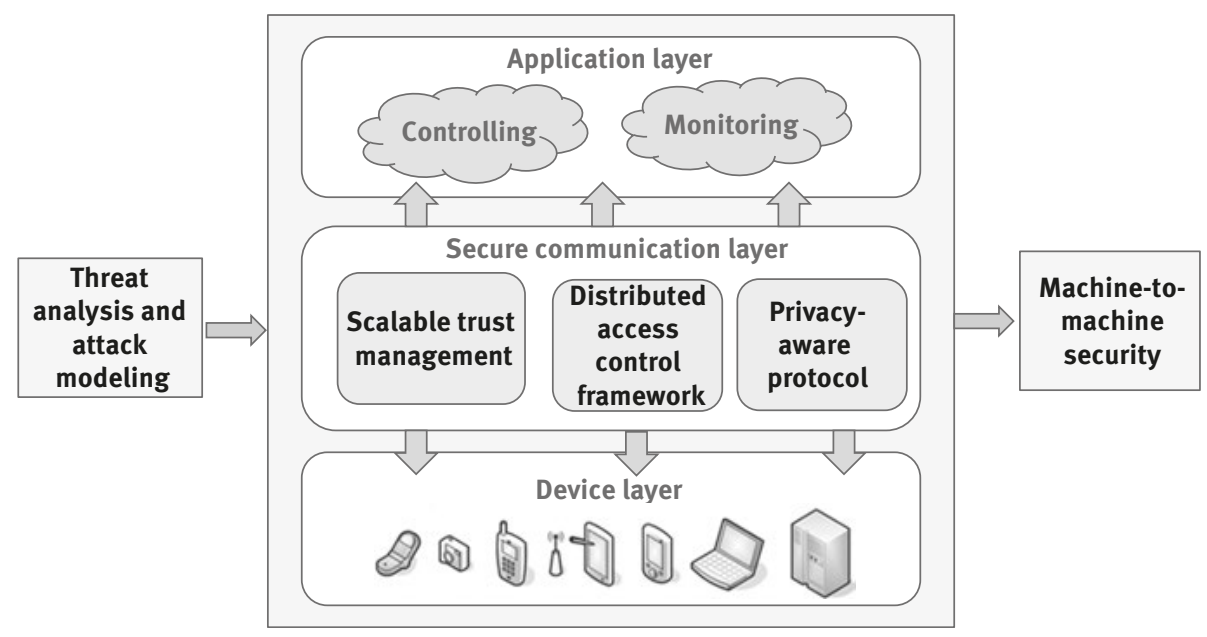

Figure 3.12: Proposed framework for secure machine to machine communication in loT.

and accuracy of results. The collected data will be forwarded to secure communication layer

\subsubsection{Secure communication layer}

This one is the middle layer that securely manages relationships between machines and services. This layer basically consists of three stages: scalable trust management model for M2M, distributed access control scheme for constrained environment and privacy-aware protocol based on the principle of least privilege. The responsibility of this layer is to carry the data that is received from device layer to cloud or application layer. To design scalable, attack-resistant communication layer we need to do critical analysis of each threat and its behavior in real-time scenario. The objective is to provide communication security without losing privacy of user.

\subsubsection{Application layer}

This layer can support many applications, for example, autonomous controlling, managing small to high-end devices to provide and perform some services and applications. Whatever data received from secure communication layer, management of that data and its suitable representation is the responsibility of application layer.

The proposed framework is a generic framework and a distributed architecture by proposing attack-resistant access control scheme, trust management scheme as well as the privacy-aware protocol for M2M in IoT. 


\subsection{Conclusion and future work}

The exponential growth of IoT in various domains in last few years leads to several threats and attacks. Unfortunately, IoT security has never been the central point of concern to researcher and developers. This chapter briefly discusses the threat analysis along with attack modeling in IoT network, and also presents a comparative analysis of various techniques that are required to secure IoT communication. The layerwise protocols along with possible attacks and threats are also presented in this chapter. The comprehensive gap analysis of existing mechanisms with respect to various attacks is presented. This chapter elaborated the requirement that must be fulfilled to make IoT secure.

In future, more efforts will be made to find out an optimum solution that resists multiple attacks at a time and to find out new opportunities in the field of IoT. In near future there is a need to develop lightweight algorithms that work in IoT network and prevent multiple attacks.

\section{References}

[1] Ashton, Kevin. “That 'Internet of things' thing”, RFID Journal, 22(7), 2009, pp. 97-114.

[2] Manyika, J., Chui, M., Bughin, J., Dobbs, R., Bisson, P. and Marrs, A., Disruptive Technologies: Advances that Will Transform Life, Business, and the Global Economy, San Francisco, CA : McKinsey Global Institute, 2013.

[3] Ren, Zejun, et al. "Security and privacy on Internet of things." Electronics Information and Emergency Communication (ICEIEC), 2017 7th IEEE International Conference on. IEEE, 2017.

[4] Madakam, S., Ramaswamy, R., and Tripathi, S.. Internet of Things (IoT): A literature review, Journal of Computer and Communications, 3(05), (2015), pp. 164.

[5] Mahalle, Parikshit Narendra, and Poonam N. Railkar. "Identity management for Internet of things”, River Publishers, 9220 Aalborg Denmark, Volume 39, ISBN: 978-87-93102-90-3 (Hard Copy) 978-87-93102-91-0 (Ebook), 2015.

[6] Lee, Suk Kyu, Bae, Mungyu, and Kim, Hwangnam. "Future of loT networks: A survey”, Applied Sciences, 7(10), 2017, pp. 1072.

[7] Al-Karaki, J. N., Chen, K. C., Morabito, G., \& De Oliveira, J. "From M2M communications to the Internet of Things: Opportunities and challenges", Ad Hoc Networks, (18), pp. 1-2. Journal ISSN : 1570-8705, DOI:10.1016/j.adhoc.2014.03.006, 2014.

[8] Mahamure, S., Railkar, P. N., and Mahalle, N. Communication protocol and queueing theorybased modelling for the Internet of Things, Journal ICT, 3, 2016, pp. 157-176.

[9] Lee, P. Y., Yu, C. M., Dargahi, T., Conti, M., and Bianchi, G. MDSClone: multidimensional scaling aided clone detection in Internet of Things, IEEE Transactions on Information Forensics and Security, 13(8), 2018, pp.2031-2046.

[10] Chugh, K., Aboubaker, L., \& Loo, J. (2012, August). "Case study of a black hole attack on LoWPAN-RPL" In Proc. of the Sixth International Conference on Emerging Security Information, Systems and Technologies (SECURWARE), Rome, Italy (pp. 157-162). Copyright (c) IARIA, 2012. ISBN: 978-1-61208-209-7, August 2012.

[11] Li, X., Lu, R., Liang, X., Shen, X., Chen, J., and Lin, X. Smart community: an Internet of things application, IEEE Communications Magazine, 49(11), 2011. 
[12] Sehgal, A., Perelman, V., Kuryla, S., and Schonwalder, J. Management of resource constrained devices in the Internet of things, IEEE Communications Magazine, 50(12), 2012.

[13] Mahamure, S., Railkar, P. N., and Mahalle, P. N. Mathematical Representation of Quality of Service (QoS) Parameters for Internet of Things (IoT), International Journal of Rough Sets and Data Analysis (IJRSDA), 4(3), 2017, pp. 96-107.

[14] Vidalis, S., and Angelopoulou, O. (2014). Assessing identity theft in the Internet of Things. Journal of IT Convergence Practice. , Vol. 2 (1): 15-21, March 2014.

[15] Simpson, A. K., Roesner, F., and Kohno, T. (2017, March). Securing vulnerable home loT devices with an in-hub security manager, In Pervasive Computing and Communications Workshops (PerCom Workshops), 2017 IEEE International Conference on (pp. 551-556). IEEE.

[16] Abu-Elkheir M., Hayajneh M., Ali NA. Data management for the Internet of Things: Design primitives and solution, Sensors. 13(11), 2013, pp.15582-15612.

[17] Gupta, B. B., Tewari, A., Jain, A. K., and Agrawal, D. P. Fighting against phishing attacks: state of the art and future challenges, Neural Computing and Applications, 28(12) 2017, pp.3629-3654.

[18] Yaqoob, I., Ahmed, E., ur Rehman, M. H., Ahmed, A. I. A., Al-garadi, M. A., Imran, M., and Guizani, $M$. The rise of ransomware and emerging security challenges in the Internet of Things, Computer Networks, 129, 2017, pp. 444-458.

[19] Bertino, E., and Islam, N. Botnets and Internet of things security. Computer, (2), 2017, pp. 76-79.

[20] Borgohain, T., Kumar, U., and Sanyal, S. Survey of security and privacy issues of Internet of things, arXiv preprint, arXiv:1501.02211, Advanced Networking and Applications Volume: 6 Issue: 4 Pages: 2372-2378 (2015) ISSN: 0975-0290, 2015.

[21] Liu, C., Yang, C., Zhang, X., and Chen, J. External integrity verification for outsourced big data in cloud and IoT: A big picture, Future generation computer systems, 49, 2015, pp. 58-67

[22] Parno, B., Perrig, A., \& Gligor, V., May). Distributed detection of node replication attacks in sensor networks, In Security and Privacy, 2005 IEEE Symposium on (pp. 49-63). IEEE, 2005.

[23] Agah, A., \& Das, S. K. Preventing DoS attacks in wireless sensor networks: A repeated game theory approach, IJ Network Security, 5(2), 2007, pp. 145-153.

[24] https://en.wikipedia.org/wiki/Replay_attack.

[25] Conti, M., Dragoni, N., and Lesyk, V. A survey of man in the middle attacks, IEEE Communications Surveys \& Tutorials, 18(3), 2016, pp. 2027-2051.

[26] Sajid, A., Abbas, H., and Saleem, K. Cloud-assisted loT-based SCADA systems security: A review of the state of the art and future challenges, IEEE Access, 4,2016, pp.1375-1384.

[27] Azmoodeh, A., Dehghantanha, A., Conti, M., and Choo, K. K. R. Detecting crypto-ransomware in loT networks based on energy consumption footprint, Journal of Ambient Intelligence and Humanized Computing, Volume 9, Issue 4, pp 1141-1152, August 2018.

[28] Kaur, Damandeep, and P. Singh, "Various OSI layer attacks and countermeasure to enhance the performance of WSNs during wormhole attack",International Journal on Network Security 5(1) 2014, pp. 62.

[29] Reed, Damon, "Applying the OSI seven layer network model to information security", SANS GIAC GSEC Practical Assignment Version 1.4 b Option One 2003.

[30] Mitrokotsa, A., Rieback, M. R., and Tanenbaum, A. S., "Classification of RFID attacks", Gen, 15693 2010, Volume 12, Issue 5, pp 491-505, 2010.

[31] Li, H., Chen, Y. and He, Z., "The survey of RFID attacks and defenses," 8th International Conference on Wireless Communications, Shanghai : Networking and Mobile Computing (WiCOM), 2012.

[32] J. Deogirikar and A. Vidhate, "Security attacks in IoT: A survey," 2017 International Conference on I-SMAC (IoT in Social, Mobile, Analytics and Cloud) (I-SMAC), Palladam, 2017, pp. 32-37, doi: 10.1109/I-SMAC.2017.8058363, 2017. 
[33] Barki, Amira, et al. "M2M security: Challenges and solutions", IEEE Communications Surveys \& Tutorials 18(2), 2016, pp. 1241-1254.

[34] Sicari, S., Rizzardi, A., Grieco, L., and Coen-Porisini, A. "Security, privacy and trust in Internet of Things The road ahead," Computer Network, 76, pp. 146-164, Jan. 2015.

[35] Firdous, Syed Naeem, et al. "Modelling and evaluation of malicious attacks against the IoT MQTT protocol." Internet of Things (iThings) and IEEE green computing and communications (GreenCom) and IEEE cyber, Physical and Social Computing (CPSCom) and IEEE Smart Data (SmartData), 2017 IEEE International Conference on. IEEE, 2017.

[36] Abdullahi Arabo, Ian Brown, and Fadi El-Moussa. Privacy in the age of mobility and smart devices in smart homes. In Privacy, Security, Risk and Trust (PASSAT), 2012 International Conference on and 2012 International Conference on Social Computing (SocialCom). IEEE, 2012, pp. 819-826.

[37] Qiu, Yue, and Maode Ma. "A mutual authentication and key establishment scheme for $\mathrm{m} 2 \mathrm{~m}$ communication in 6lowpan networks", IEEE Transactions on Industrial Informatics, 12(6) 2016, pp.2074-2085.

[38] Y. Lin, J. Huang, C. Fan and W. Chen, "Local Authentication and Access Control Scheme in M2M Communications With Computation Offloading," in IEEE Internet of Things Journal, vol. 5, no. 4, pp. 3209-3219, Aug. 2018.

[39] Mahalle, Parikshit, et al. "Identity establishment and capability based access control (IECAC) scheme for Internet of Things.” WPMC. 2012.

[40] Mahalle, P. N., Anggorojati, B., Prasad, N. R., and Prasad, R. Identity authentication and capability based access control (iacac) for the Internet of things, Journal of Cyber Security and Mobility, 1(4), 2013, pp. 309-348.

[41] A. Esfahani et al., "A Lightweight Authentication Mechanism for M2M Communications in Industrial IoT Environment," in IEEE Internet of Things Journal, vol. 6, no. 1, pp. 288-296, doi: 10.1109/JIOT.2017.2737630, Feb. 2019.

[42] Chen, Hsing-Chung, et al. "A security gateway application for End-to-End M2M communications," Computer Standards \& Interfaces, 44 2016, pp. 85-93.

[43] Mahalle, P., Babar, S., Prasad, N. R., and Prasad, R. (July). Identity management framework towards Internet of things (IoT): Roadmap and key challenges, In International Conference on Network Security and Applications (pp. 430-439), Berlin, Heidelberg: Springer, 2010.

[44] Dey, N., Hassanien, A. E., Bhatt, C., Ashour, A., and Satapathy, S. C. (Eds.). Internet of things and big data analytics toward next-generation intelligence, Berlin: Springer, 2018.

[45] Elhayatmy G., Dey N., Ashour A.S. (2018) Internet of Things Based Wireless Body Area Network in Healthcare. In: Dey N., Hassanien A., Bhatt C., Ashour A., Satapathy S. (eds) Internet of Things and Big Data Analytics Toward Next-Generation Intelligence. Studies in Big Data, vol 30. Springer, Cham.

[46] Bhatt, C., Dey, N., and Ashour, A. S. (Eds.). Internet of things and big data technologies for next generation healthcare (Vol. 23). New York: Springer, 2017.

[47] Dey N., Ashour A.S., Bhatt C. (2017) Internet of Things Driven Connected Healthcare. In: Bhatt C., Dey N., Ashour A. (eds) Internet of Things and Big Data Technologies for Next Generation Healthcare. Studies in Big Data, vol 23. Springer, Cham

[48] Hassanien, A. E., Dey, N., and Borra, S. (Eds.). (2018). Medical Big Data and Internet of Medical Things: Advances, Challenges and Applications. CRC Press, Pub. location Boca Raton, eBook ISBN 9781351030380, 2018.

[49] Sarowar, M. G., Kamal, M. S., and Dey, N. (2019). Internet of Things and Its Impacts in Computing Intelligence: A Comprehensive Review - IoT Application for Big Data. In N. Dey, and S. Tamane (Eds.), Big Data Analytics for Smart and Connected Cities (pp. 103-136). Hershey, PA: IGI Global. doi:10.4018/978-1-5225-6207-8.ch005 
[50] Kimbahune, V. V., Deshpande, A. V., and Mahalle, P. N. Lightweight key management for adaptive addressing in next generation internet. International Journal of Ambient Computing and Intelligence (IJACI), 8(1), 2017, pp.50-69.

[51] Parne, B. L., Gupta, S., and Chaudhari, N. S. Segb: Security enhanced group based aka protocol for $\mathrm{m} 2 \mathrm{~m}$ communication in an IoT enabled LTE/LTE - a network, IEEE Access, 6 , 2018, pp. 3668-3684.

[52] Wang, K., Du, M., Sun, Y., Vinel, A., and Zhang, Y. Attack detection and distributed forensics in machine-to-machine networks, IEEE Network, 30(6), 2016, pp. 49-55.

[53] Broustis, I., Sundaram, G. S., \& Viswanathan, H. Detecting and preventing machine-tomachine hijacking attacks in cellular networks, Bell Labs Technical Journal, 17(1), 2012, pp. 125-140.

[54] Doh, I., Lim, J., Li, S., and Chae, K. July). Key establishment and management for secure cellular machine-to-machine communication. In 2013 Seventh International Conference on Innovative Mobile and Internet Services in Ubiquitous Computing (IMIS), 2013, (pp. 579-584). IEEE.

[55] Purohit, Kamlesh C., et al. "Hybrid approach for securing loT communication using authentication and data confidentiality." 2017 3rd International Conference on Advances in Computing, Communication \& Automation (ICACCA)(Fall). IEEE, 2017. 